\title{
Apoyo social para la parentalidad: experiencias en programas de infancia en Chile ${ }^{1}$
}

\author{
Lilian Sanhueza Díaz², Javiera Saldías³, \\ Yasna Contreras ${ }^{4}$, Paola Pizarro ${ }^{5}$
}

\begin{abstract}
El artículo da cuenta de las principales dificultades que enfrentan las figuras parentales atendidas en programas de protección de derechos de la infancia en la región de la Araucanía, al sur de Chile, y los apoyos disponibles en los ámbitos emocional, informativo e instrumental. Mediante una investigación de tipo cualitativo y cuantitativo, se encuestó a 99 padres, madres o cuidadores, y se aplicó una entrevista en profundidad a 6 madres o cuidadoras pertenecientes a distintas configuraciones familiares y procedencia étnica y territorial. Se constató que quienes participan en los procesos de intervención son mayoritariamente mujeres - madres o cuidadoras- que enfrentan diversas dificultades emocionales, instrumentales e informativas, cuentan con escasas redes informales de apoyo y el sustento social que reciben de las redes formales tiende a deteriorar su autoeficacia parental.
\end{abstract}

1 Este artículo de investigación científica es parte de la investigación "Modelo de Evaluación de Condiciones para la Parentalidad" (MECeP), realizada en el marco de la Política de Protección de Derechos de la Infancia, financiada por el Fondo de Fomento al Desarrollo Científico y Tecnológico (FONDEF) del Gobierno de Chile y desarrollada entre diciembre de 2011 y noviembre de 2013. La discusión de resultados se ha nutrido de las reflexiones del grupo Interdisciplinario en Estudios de Género de la Universidad Católica de Temuco.

2 Trabajadora Social del Departamento de Trabajo Social, Facultad de Ciencias Sociales, Universidad Católica de Temuco. Chile. Académica en el Magíster en Trabajo Social y Magíster en Resolución Colaborativa de Conflictos de las facultades de Ciencias Sociales y Humanidades, y de la Facultad de Ciencias Jurídicas, respectivamente, en la Universidad Católica de Temuco. Investigadora y directora alterna proyecto FONDEF Idea CA12i10076. Miembro Grupo Interdisciplinario en Estudios de Género de la Universidad Católica de Temuco. E-mail: 1sanhueza@uct.cl.

3 Egresada del Departamento de Trabajo Social, Facultad de Ciencias Sociales, Universidad Católica de Temuco, Chile. Tesista en el marco de proyecto FONDEF Idea CA12i10076. E-mail: javiera.saldia@gmail.com

4 Egresada del Departamento de Trabajo Social, Facultad de Ciencias Sociales, Universidad Católica de Temuco, Chile. Tesista en el marco de proyecto FONDEF Idea CA12i10076. E-mail: ycontrerasfoitzick@gmail.com

5 Egresada del Departamento de Trabajo Social, Facultad de Ciencias Sociales, Universidad Católica de Temuco, Chile. Tesista en el marco de proyecto FONDEF Idea CA12i10076. E-mail: ppizarro2009@alu.uct 
Apoyo social para la parentalidad: experiencias en programas de infancia en Chile / Sanhueza et al.

Palabras clave: sistema de protección infantil, trabajo social con familias, género, apoyo social formal e informal, autoeficacia parental

\section{Social Support for Parenthood: experiences in children's programs in Chile}

This article reports on the principal difficulties faced by parental figures involved with programs for protection of childhood rights in Araucanía Region, Southern Chile, and support mechanisms available for emotional, informational, and instrumental aid. A qualitative and quantitative research methodology was used to survey 99 parents and guardians, with indepth interviews for 6 mothers or guardians belonging to different family configurations, from different ethnic origins and areas. It was found that most people involved in intervention processes are women - mothers or guardians - who face a wide range of emotional, instrumental, and information difficulties, possess limited informal support networks, and receive social intervention from formal networks that tends to only impair their parental self-effectiveness.

Keywords: child protection system, social work with families, gender, formal and informal social support, parental self-effectiveness

\section{Apoio Social para a parentalidade: experiências em programas de infância no Chile}

RESUMO

$\mathrm{O}$ artigo expõe as principais dificuldades enfrentadas pelas figuras parentais atendidas em programas de proteção de direitos da infância na região da Araucanía, no sul do Chile, e o apoio disponível nos âmbitos emocional, informativo e instrumental. Através de uma investigação de tipo qualitativo e quantitativo, foram entrevistados 99 pais, mães ou cuidadores e aplicou-se uma entrevista aprofundada a 6 mães ou cuidadoras pertencentes a distintas configurações familiares e procedência étnica e territorial. Constatou-se que quem participa dos processos de intervenção são principalmente mulheres -mães ou cuidadoras- que enfrentam diversas dificuldades emocionais, instrumentais e informativas, contam com escassas redes informais de apoio e a sustentação social que recebem das redes formais tende a deteriorar sua autoeficácia parental.

Palavras-chave: sistema de proteção infantil, trabalho social com famílias, gênero, apoio social formal e informal, autoeficácia parental 


\section{Introducción}

A partir de la suscripción de la Convención Internacional por los Derechos del Niño, en 1990, Chile se compromete a ejecutar una serie de medidas, tanto a nivel legislativo como de política pública, orientadas a garantizar el bienestar y los derechos de niños, niñas y adolescentes. La entidad gubernamental encargada de liderar la política de infancia en el país y orientar a los organismos colaboradores es el Servicio Nacional de Menores (SENAME). Frente al riesgo o a la evidencia de situaciones de vulneración de derechos de la infancia, existe una serie de programas de protección de derechos, en su mayoría implementados por los organismos colaborados - corporaciones, fundaciones u otros de origen privado-, que guían su intervención a partir de las orientaciones y bases técnicas que periódicamente define el SENAME. Si bien es cierto el principal sujeto de atención en estos programas es el niño, niña y/o adolescente, las bases técnicas plantean la relevancia de trabajar con las familias y la comunidad. Específicamente, el apoyo a las familias constituye una de las recomendaciones del Comité de los Derechos del Niño (2007), en la línea de garantizar los derechos de niños y niñas a través del fortalecimiento de las familias y sus entornos.

Muchas de las familias atendidas en programas de protección de derechos de la infancia se encuentran en situación de vulnerabilidad y exclusión social, por lo que la activación de sus redes de apoyo social constituye un aspecto clave a abordar. Diversos estudios de género han demostrado que el cuidado de los niños y niñas ha estado asignado histórica y culturalmente a mujeres, madres o cuidadoras. En este sentido, los estudios feministas develan la asociación naturalizada de ser mujer-madre-esposa, portadora de una representación social de la maternidad altamente idealizada, que la responsabiliza de la estabilidad familiar y social en el marco de la familia moderna (Beauvoir, 1949; Aguayo, Barker \& Kimelman, 2016; Chacón \& Tapia, 2017), lo que genera un profundo malestar psíquico en mujeres que no pueden cumplir con estas expectativas, llamado también el "malestar sin nombre" (Friedan, 1963). 
A partir del presente trabajo se busca identificar los tipos de apoyo para la parentalidad requeridos por las familias, específicamente por las figuras parentales - en su mayoría mujeres, madres y/o cuidadoras-, las redes de apoyo que estas reconocen y la valoración que hacen de ellas. Las preguntas que guiaron la investigación fueron: ¿Cuáles son las principales dificultades que enfrentan las figuras parentales? ¿Qué tipos de apoyo requieren? ¿Con qué redes de apoyo cuentan? ¿Qué valoración hacen de las redes formales e informales? ¿Cómo se relaciona el género, mayoritariamente femenino de las figuras parentales, con el apoyo social disponible?

\section{Parentalidad}

El concepto "parentalidad", en inglés parenthood, fue acuñado por la psicoanalista húngara Therese Benedek en 1959, para dar cuenta del complejo proceso psíquico de maduración que se desarrolla tanto en la madre como en el padre, por el hecho de convertirse en padres, y lo distingue de "maternidad" y "paternidad", (Cabriolé y Sanhueza, 2016). Dado su origen anglosajón, el concepto no tiene género; sin embargo, literatura reciente en el mundo hispanoparlante ha optado por distinguir entre parentalidad y marentalidad como una forma de dar cuenta de las diferencias de género presentes en las experiencias entre hombres y mujeres.

Para Cabrolié y Sanhueza (2016), la parentalidad es una relación entre un adulto reconocido como madre o padre por un niño(a) o adolescente, que se construye a lo largo de la vida mediante un vínculo principalmente afectivo y simbólico, más que biológico o legal. Esta relación emerge al interior de un contexto cultural, en una época y en un marco jurídico particular. Las autoras consideran que la parentalidad es básicamente una relación en la que interactúan, al menos, un adulto(a) y un niño(a); relación que puede verse afectada por las condiciones del contexto sociocultural, de los padres, madres o cuidadores(as), e incluso, de los propios niños y niñas. Dentro del contexto sociocultural, se encuentra el apoyo social. 
Las condiciones del contexto sociocultural son entendidas como "los aspectos del entorno familiar, social y cultural, estructurales y simbólicos (...) respecto de los cuales los padres, madres y/o cuidadores tienen escasas posibilidades de incidir o transformar" (Cabriolé y Sanhueza, 2016), que pueden afectar positiva o negativamente en la parentalidad. En este sentido, Rodrigo, Márquez y Martin (2010) también mencionan la relevancia del contexto sociocultural, señalando que "la tarea de ser padres y madres no se ejerce en un vacío ni depende exclusivamente de las características de los progenitores. Se ejerce dentro de un espacio ecológico" (p. 13).

De este modo, las diversas dificultades para la parentalidad requieren de apoyos que faciliten la tarea de cuidado y crianza de los niños, en especial a aquellas familias más vulnerables. En este sentido, un estudio chileno muestra que las madres en familias monoparentales —en su mayoría encabezadas por mujeres jefas de hogar- presentan niveles de estrés significativamente más altos que las madres de familias nucleares (Olhaberry \& Farkas, 2012).

La vinculación entre apoyo social y parentalidad fue planteada por Rodrigo (2009) en un estudio con familias atendidas en programas de servicios sociales municipales en situación de riesgo psicosocial, en Islas Canarias, España. De acuerdo con el estudio, las familias en riesgo psicosocial son aquellas que afrontan mayores dificultades en su vida cotidiana, las que pueden tener un impacto negativo y deteriorar la relación parental, al demandar a los padres mayor atención en la búsqueda de soluciones a estas dificultades que a las necesidades de sus hijos e hijas. 
Apoyo social para la parentalidad: experiencias en programas de infancia en Chile / Sanhueza et al.

Tabla 1: Tipo y fuentes de apoyo social en madres según nivel de riesgo psicosocial.

\begin{tabular}{|c|c|c|}
\hline Nivel de riesgo & Tipo de apoyo & Fuentes de apoyo \\
\hline Sin riesgo & $\begin{array}{l}\text { Ambas fuentes de apoyo tanto } \\
\text { formal como informal }\end{array}$ & $\begin{array}{l}\text { Colegio } \\
\text { Pareja }\end{array}$ \\
\hline Bajo riesgo & $\begin{array}{l}\text { Primacía de fuentes de apoyo } \\
\text { informal }\end{array}$ & $\begin{array}{l}\text { Amigos } \\
\text { Pareja } \\
\text { Vecinos } \\
\text { Escuela }\end{array}$ \\
\hline Medio-alto & $\begin{array}{l}\text { Primacía de fuentes de apoyo } \\
\text { formal }\end{array}$ & $\begin{array}{l}\text { Sanidad } \\
\text { Cruz Roja } \\
\text { Otras ONG } \\
\text { Otras personas } \\
\text { Dirección general del menor } \\
\text { Abuelos }\end{array}$ \\
\hline
\end{tabular}

Fuente: Elaboración propia con base en el texto "Una mirada integradora de la resiliencia parental: desde el contexto hasta la mente de las madres y los padres en riesgo psicosocial" (2009).

Como se puede apreciar en la tabla 1, las fuentes de apoyo a las que recurren las familias con mayor riesgo psicosocial son las redes formales, a excepción de los abuelos, quienes forman parte de la red de apoyo informal ante la que acuden las familias en situaciones de crisis (Rodrigo, 2009). La autora constató que, "a medida que se incrementa el riesgo psicosocial en la familia, ésta suele contar con más apoyos formales que informales, exceptuando aquellos casos que presentan un aislamiento social extremo con carencia tanto de fuentes formales como informales" (p. 17). Cabe destacar que el estudio de Rodrigo se centró en las madres como cuidadoras principales y no consideró otros cuidadores, como padres, abuelos u otras figuras adultas significativas. Otros estudios desarrollados en Europa con jóvenes en programas de residencia - aquellos que han debido ser separados de sus familias-, muestran que es necesario potenciar los vínculos con redes de apoyo social informal, que están presentes en la comunidad, particularmente en aquellos casos en los que resulta inviable un proceso de reunificación familiar (Bravo y Del Valle, 2003; Kendrick, 2005; Daining y de Panfilis, 2007; Martín y Dávila, 2008; Martin, 2011). 
Por otra parte, respecto de niños y niñas, diversa literatura alude a que algunas características especiales de estos(as), tales como problemas conductuales, de sueño, de aprendizaje, de alimentación o de control de esfínter, entre otros, dificultan la relación con sus padres (Díaz, Pérez, Martínez, Brito y Herrera, 2001; Correa, Rodrigo, Rodríguez y Simón, 1998). En el caso de madres de niños con trastorno de espectro autista, se ha constatado que estas presentan altos niveles de estrés parental y riesgo de depresión, efectos que se ven mitigados cuando las madres cuentan con fuentes de apoyo informal (Pozo Cabanillas, Sarriá Sánchez, Méndez Zaballos, 2006).

\section{Apoyo social y género}

De acuerdo con la teoría del apoyo social, las personas, ante situaciones de peligro, temor, ansiedad o estrés, buscan, de manera natural, la compañía de otras personas en la misma situación, lo que les permitiría validar sus propias experiencias y reacciones mediante un proceso de "comparación social" con personas que comparten una misma situación o problema (Gracia, 1997; Gallardo \& Gálvez, 2018).

Algunos estudios plantean que los sujetos sometidos a largos periodos de aislamiento experimentan diversa sintomatología psicológica (Byrd, 1939 y Lilly, 1956, citados en Gracia, 1997; Gómez, Muñoz \& Haz, 2007) y aumentan el nivel de ansiedad, lo que repercute en su salud mental. Incluso, algunas autoras señalan que la introducción del concepto de "apego", del psicoanalista John Bowlby, en 1951, aumenta la ansiedad de las madres que se ven sobreexigidas, dado el vínculo privilegiado que se espera que desarrollen con sus hijos (Burin y Meler, 2001), generando en ellas sentimientos de intimidación y culpabilización cuando ello no ocurre (Knibiehler, 2001).

Por otra parte, Cassel (1974) y Cobb (1976) comprobaron, por separado, que "los sujetos que contaban con la compañía de pares significativos o se encontraban en un contexto interactivo de apo- 
yo no mostraban los cambios negativos en salud que presentaban quienes enfrentaban situaciones difíciles y/o estresantes en condiciones de aislamiento social" (Gracia, Herrero y Musitu, 2002, p. 19). En este sentido, las mujeres, al estar relegadas al espacio doméstico, fruto del "contrato sexual" heredado de la ilustración (Pateman, 1988), ven coartadas sus posibilidades de interactuar y contar con "otras mujeres significativas", que les permitan reconstruir sus subjetividades más allá de la esfera del mundo privado. Esto es especialmente complejo cuando estas mujeres se dedican exclusivamente al cuidado de otros.

Dentro de la teoría del apoyo social destacan las perspectivas estructural y funcional (Terol, López, Neipp, Rodríguez, Pastor, Martín-Aragón, 2004). La primera analiza las características de las redes sociales y las condiciones objetivas que rodean el apoyo social. Por otra parte, la perspectiva funcional visualiza en qué medida las relaciones sociales satisfacen ciertas necesidades de los individuos y reconoce tres tipos de apoyo: emocional, instrumental e informativo. Se ha identificado que el apoyo emocional es aquel que tiene un mayor impacto en la salud mental de las personas.

Ahora bien, las fuentes de donde proviene el apoyo social pueden ser muy diversas y se distinguen aquellas formales e informales. Las redes formales incluyen a profesionales que operan basados en un sistema de categorías, reglas formales y protocolos que regulan la relación de apoyo y consideran criterios objetivos respecto de lo que constituye éxito o fracaso de la intervención (Gracia, 1997; Gracia et al., 2002; Lila y Gracia, 1996, citado en Rodrigo, 2009). Las redes informales de apoyo se caracterizan por ser de tipo horizontal, bidireccional, en las que existen al menos dos sujetos que viven experiencias similares y que pueden apoyarse mutuamente en distintas circunstancias de la vida, que ambos pueden definir libremente.

El apoyo social que brindan las redes formales se establece en el marco de un sistema de ayuda basado en una relación vertical, 
unidireccional, en la que existe un sujeto experto activo y otro sujeto ignorante-desorientado, pasivo. Por el contrario, las redes informales de apoyo no suelen menoscabar los sentimientos de competencia y control de las personas sobre sus vidas, y su uso, inclusive, incrementa estos sentimientos y potencia el compromiso, la reciprocidad y la responsabilidad por el cuidado de otros (Rodrigo, 2009). Estos sentimientos de competencia y control sobre la propia vida constituyen lo que se denomina "autoeficacia", concebida como el conjunto de creencias que tienen las personas acerca de sus capacidades para lograr determinados resultados y que influye en las expectativas y el desarrollo de comportamientos y estrategias para afrontar el estrés. La autoeficacia parental se refiere al grado en el que la persona se siente capacitada para cumplir con el rol de padre o madre, lo que determina expectativas positivas respecto de sus habilidades para ello y el grado de satisfacción con ese logro (Farkas, 2008). En el caso de familias en riesgo psicosocial, se ha determinado que el estrés parental de las madres es inversamente proporcional al grado de satisfacción que ellas sienten respecto del ejercicio del rol materno (Ayala-Nunes, Lemos \& Nunes, 2013).

Las redes formales de apoyo provienen principalmente del Estado y de las políticas públicas que definen y operan los programas mediante profesionales que se desempeñan en esa labor. De acuerdo con Meler (1998), la subjetividad se construye en un contexto sociocultural en el que disputan sentido representaciones imaginarias hegemónicas y alternativas. En este sentido, la construcción subjetiva de la maternidad en las mujeres que participan de los programas de protección a la infancia se produce en una trama de vínculos en permanente interacción. Así, el Estado, con su trabajo de codificación, define acciones y políticas que refuerzan cierta forma de organización familiar considerada legítima (Bourdieu, 1997). Los programas gubernamentales se basan en modelos familiares con estructuras y relaciones de género que no siempre corresponden a la realidad social y que invisibilizan las transformaciones que ocurren al interior de las familias (García y de Oliveira, 2011). 
Apoyo social para la parentalidad: experiencias en programas de infancia en Chile / Sanhueza et al.

\section{Metodología}

\section{Muestra}

Se consideró una metodología de tipo mixta, cuantitativa y cualitativa. Se trabajó con una muestra de 99 adultos: padres, madres o cuidadores a cargo de niños y niñas que participaban en programas de protección de derechos de infancia, de un universo de 272 adultos atendidos en 5 programas de distintos territorios de la Región de la Araucanía, al sur de Chile, durante 2014.

Tabla 2: Familias participantes por programa.

\begin{tabular}{|l|c|c|c|}
\hline \multicolumn{1}{|c|}{ Programa } & $\begin{array}{c}\text { Cobertura } \\
\text { familias } \\
\text { atendidas }\end{array}$ & $\begin{array}{c}\text { Muestra } \\
\text { familias por } \\
\text { estrato }\end{array}$ & $\begin{array}{c}\text { Porcentaje } \\
\text { muestra }\end{array}$ \\
\hline $\begin{array}{l}\text { Programa familia de acogida } \\
\text { simple (FAS) Villarrica }\end{array}$ & 50 & 18 & $18 \%$ \\
\hline $\begin{array}{l}\text { Programa de intervención } \\
\text { breve (PIB) Victoria }\end{array}$ & 75 & 28 & $28 \%$ \\
\hline $\begin{array}{l}\text { Programa de intervención } \\
\text { especializada (PIE) Villarrica }\end{array}$ & 50 & 18 & $18 \%$ \\
\hline $\begin{array}{l}\text { Programa familia de acogida } \\
\text { especializada (FAE) Temuco }\end{array}$ & 42 & 15 & $15 \%$ \\
\hline $\begin{array}{l}\text { Programa familias de acogida } \\
\text { simple (FAS) }\end{array}$ & 55 & 20 & $20 \%$ \\
\hline Total & 272 & 99 & $100 \%$ \\
\hline
\end{tabular}

Fuente: Elaboración propia.

La muestra presentó un margen de error de un $8 \%$ y se aplicó una escala tipo Likert que consideraba cuatro ítems: 1) antecedentes familiares; 2) necesidades de apoyo y tipos de apoyo social disponible: emocional, instrumental e informativo; 3) redes de apoyo y valoración de estas; 4) sugerencias y comentarios cualitativos. Para analizar los datos, se utilizó el software SPSS. La encuesta fue validada mediante un proceso previo de pilotaje en dos familias de programas de protección de infancia, con similares características a la población objeto de este estudio. 
Se realizó una entrevista en profundidad a 6 familias de diferente configuración y procedencia étnica y territorial, en la que se abordaron tres tópicos: dificultades en torno a la parentalidad, apoyo social requerido y redes de apoyo social disponibles. Con ello se buscó reconstruir las experiencias particulares de las madres desde una perspectiva comprensiva y fenomenológica (Schütz, 2003; Berger \& Luckmann, 2008).

Tabla 3: Participantes de entrevista en profundidad.

\begin{tabular}{|l|l|}
\hline Configuración familiar & Programa en el que participa \\
\hline Familia monoparental & Programa familia acogida simple (FAS) Villarrica. \\
\hline Familia reconstituida & Programa intervención especializada (PIE) Villarrica. \\
\hline Familia nuclear biparental & Programa familia acogida simple (FAS) Temuco. \\
\hline Familia extensa & Programa familia acogida especializada (FAE) Temuco. \\
\hline Familia no mapuche rural & Programa intervención breve (PIB) Temuco. \\
\hline Familia mapuche rural & Programa de intervención breve (PIB) Temuco. \\
\hline
\end{tabular}

Fuente: Elaboración propia.

La información recogida fue categorizada e interpretada mediante análisis de contenido, en la lógica de la teoría fundamentada (Strauss y Corbin, 2002). Los pasos fueron: 1) transcripción literal de las entrevistas; 2) selección y segmentación de los textos escritos, en los que se identificaba pasajes relevantes acordes con los objetivos planteados para el estudio; 3) identificación de los códigos manifiestos presentes en el relato de las personas; 4) identificación de las categorías semánticas significativas presentes en los textos analizados; 5) agrupación de las categorías por tipo, y 6) análisis de las categorías obtenidas acordes con el enfoque adoptado para este estudio (Bardin, 1986; Navarro y Díaz, 1995, Piñuel, 2002). Cada una de los participantes firmó un consentimiento ético informado que garantiza la confidencialidad y fines académicos de la información, en consonancia con lo establecido en los principios de la Declaración de Singapur sobre la integridad en la investigación. 


\section{Resultados y discusión}

\section{Antecedentes familiares}

Un primer resultado interesante es la presencia mayoritaria de mujeres como principales figuras parentales responsables de niños y niñas atendidos en los programas de protección de derechos de la infancia en la Araucanía. Más del 85\% de quienes contestaron la encuesta, en su rol como "adultos responsables", fueron mujeres - madres, tías, abuelas, nuevas parejas del padre, entre otras-.

Esto demuestra que los procesos de intervención psicosocial continúan privilegiando la participación de mujeres en estos programas y perpetuando su rol de cuidadoras, en detrimento de la incorporación de figuras masculinas. Menos de un 15\% de los encuestados fueron varones: padres o abuelos.

\section{Dificultades parentales y apoyo social disponible}

Los encuestados señalan dificultades para la parentalidad en las tres áreas del apoyo social: instrumental, emocional e informativo. Más de la mitad $(55,21 \%)$ ha enfrentado dificultades en la crianza de sus hijos por razones económicas, por lo que requieren apoyo instrumental, y solo un $58,16 \%$ de los encuestados señala contar con alguien que cuide de sus hijos ante una situación de emergencia. Más de dos tercios $(72,16 \%)$ se ha sentido frustrado o angustiado y más de la mitad señala dificultades para establecer normas o límites a sus hijos (55,67\%), que se enmarcan en el ámbito emocional. Finalmente, en cuanto al apoyo informativo, se aprecia que más de un tercio ha tenido dudas acerca de cómo enfrentar situaciones difíciles en la crianza $(68,04 \%)$ y más de la mitad indica que ha requerido información acerca de enfermedades que han afectado a sus hijos $(62,24 \%)$ y ha tenido problemas para apoyarlos en las tareas escolares $(57,58 \%)$. 
Tabla 4: Dificultades parentales según tipo de apoyo social requerido.

\begin{tabular}{|l|l|c|}
\hline \multicolumn{1}{|c|}{ Tipo de apoyo } & \multicolumn{1}{|c|}{ Afirmaciones } & Porcentaje \\
\hline Apoyo instrumental & $\begin{array}{l}\text { He enfrentado problemas económicos que } \\
\text { me han hecho difícil el criar a mis hijos/as. }\end{array}$ & $55,21 \%$ \\
\cline { 2 - 3 } & $\begin{array}{l}\text { Ante una emergencia tendría con quien dejar } \\
\text { a mis hijos. }\end{array}$ & $58,16 \%$ \\
\hline Apoyo emocional & $\begin{array}{l}\text { Es difícil para mí establecer normas a mis } \\
\text { hijos/as. }\end{array}$ & $55,67 \%$ \\
\cline { 2 - 4 } & $\begin{array}{l}\text { A veces siento que no soy claro con las nor- } \\
\text { mas que pongo a mis hijos y necesito que } \\
\text { alguien me oriente. }\end{array}$ & $62,89 \%$ \\
\cline { 2 - 4 } & $\begin{array}{l}\text { Me he sentido sobrepasado/a en la crianza de } \\
\text { mis hijos/as. }\end{array}$ & $53,68 \%$ \\
\cline { 2 - 4 } & $\begin{array}{l}\text { Me he sentido enojado/a mucho tiempo por } \\
\text { el comportamiento de mis hijos/as. }\end{array}$ & $35,42 \%$ \\
\hline Apoyo informativo & $\begin{array}{l}\text { He tenido dudas acerca de cómo enfrentar } \\
\text { situaciones difíciles en la crianza. }\end{array}$ & $68,04 \%$ \\
\cline { 2 - 4 } & $\begin{array}{l}\text { Hay situaciones en las que no sé cómo con- } \\
\text { trolar el comportamiento de mi hijo/a. }\end{array}$ & $62,24 \%$ \\
\cline { 2 - 3 } & $\begin{array}{l}\text { He necesitado información frente a enferme- } \\
\text { dades que han afectado a mis hijos/as. }\end{array}$ & $62,24 \%$ \\
\hline & $\begin{array}{l}\text { Me cuesta ayudar a mis hijos/as en las tareas } \\
\text { del colegio. }\end{array}$ & $57,58 \%$ \\
\hline
\end{tabular}

Fuente: Elaboración propia.

Los padres identifican redes de apoyo formales e informales, y destacan las instituciones del Estado en un 78,13\% de los casos. Un 68,75\% reconoce recibir beneficios del Estado para solventar los gastos de sus hijos y solo un $39,58 \%$ acude a su familia cuando no tiene el dinero suficiente para satisfacer las necesidades básicas de sus hijos. Sin embargo, ante necesidades económicas, un $39,80 \%$ preferiría acudir a una institución o persona externa a la familia. Un 52,04\% acude a redes informales, como familia o amigos, para recibir apoyo emocional -básicamente contención respecto de problemas que viven en el proceso de crianza- y solo un $30,93 \%$ señala que sus amigos han sido fundamentales como apoyo parental. Existe una escasa valoración del apoyo que otras or- 
Apoyo social para la parentalidad: experiencias en programas de infancia en Chile / Sanhueza et al.

ganizaciones informales pueden brindar (39,58\%) y cabe destacar que solo el 52,58\% de las encuestadas informa contar con apoyo económico del padre de su hijo.

Tabla 5: Redes de apoyo social disponibles para la parentalidad.

\begin{tabular}{|c|c|c|}
\hline Redes de apoyo & Afirmaciones & Porcentaje \\
\hline \multirow{5}{*}{ Formal } & $\begin{array}{l}\text { Puedo contar con las instituciones del Estado } \\
\text { cuando existe una situación que afecta a mis hi- } \\
\text { jos/as. }\end{array}$ & $78,13 \%$ \\
\hline & $\begin{array}{l}\text { Si necesitara dinero para satisfacer las necesi- } \\
\text { dades de mis hijos/as, preferiría acudir a alguna } \\
\text { institución o persona externa que a los miembros } \\
\text { de mi familia. }\end{array}$ & $39,80 \%$ \\
\hline & $\begin{array}{l}\text { Los programas de protección a la infancia me } \\
\text { apoyan económicamente para la manutención de } \\
\text { mi hijo. }\end{array}$ & $35,35 \%$ \\
\hline & $\begin{array}{l}\text { He participado en comités, organizaciones so- } \\
\text { ciales, iglesia, etc., que me han ayudado en la } \\
\text { crianza. }\end{array}$ & $39,58 \%$ \\
\hline & $\begin{array}{l}\text { Recibo actualmente beneficios del Estado que me } \\
\text { permiten solventar gastos de mis hijos/as. }\end{array}$ & $68,75 \%$ \\
\hline \multirow{5}{*}{ Informal } & $\begin{array}{l}\text { Cuando necesito desahogarme o contar los pro- } \\
\text { blemas que he tenido para criar a mis hijos/as, } \\
\text { acudo a mi familia o amigos. }\end{array}$ & $52,04 \%$ \\
\hline & $\begin{array}{l}\text { Si enfrentara una situación de desempleo tendría } \\
\text { familiares y amigos que me brinden apoyo. }\end{array}$ & $72,45 \%$ \\
\hline & $\begin{array}{l}\text { Tengo el apoyo del padre de mis hijos/as para } \\
\text { solventar los gastos materiales que demanda la } \\
\text { crianza. }\end{array}$ & $52,58 \%$ \\
\hline & $\begin{array}{l}\text { Mis amigos son fundamentales en la crianza de } \\
\text { mis hijos/as, ya que me entregan consejos y siem- } \\
\text { pre están disponibles ante cualquier situación. }\end{array}$ & $30,93 \%$ \\
\hline & $\begin{array}{l}\text { Cuando no tengo el dinero necesario para satisfa- } \\
\text { cer las necesidades básicas de mis hijos/as, acudo } \\
\text { a mi familia. }\end{array}$ & $39,58 \%$ \\
\hline
\end{tabular}

Fuente: Elaboración propia.

En términos cualitativos, las madres o cuidadoras señalan dificultades en relación con el establecimiento de normas y límites: 
Poner reglas (...) de primera era caótica la cosa, el niño... traía su forma, estos otros estaban criados de distinta forma, no fue fácil porque traía unas mañitas grandes totalmente diferentes a mis hijos (...); en el colegio, al que tiene doce años le va bien, al de diez también, pero a la niñita que es la única mujer es media floja para estudiar, y el otro más grande nos ha complicado mucho más porque resulta que el entró en una depresión y es más complicado... (Familia extensa).

Por otra parte, la tarea de establecer normas y límites se asocia con la figura parental masculina, desde estereotipos de género que atribuyen a la madre un papel más emocional vinculado al afecto, $\mathrm{y}$ al padre un rol de autoridad asociado a la capacidad de establecer normas y límites:

Porque yo tengo mi nueva pareja, entonces, ahí tiene un respeto, hay un límite en ese tipo de cosas (...); mi esposo, porque yo me casé nuevamente, él es la única persona que lo hace aterrizar. (Familia reconstituida)

La falta de apoyo de la pareja es especialmente significativa para las familias monoparentales, particularmente cuando están en aislamiento social, como es el caso de familias rurales. Aun cuando la estructura familiar en sí misma no constituye riesgo, se ha estudiado que la monoparentalidad puede ser una situación crítica cuando se accede a ella de manera involuntaria, y no se cuenta con las redes de apoyo necesarias para desarrollar las múltiples tareas que implica conciliar la parentalidad con el trabajo y otros aspectos de la vida personal (Jociles, Rivas, Moncó, Villamil y Díaz, 2008). Así, la falta de fuentes de apoyo social incide en la sensación de soledad que experimentan las madres y cuidadoras:

Por ejemplo, para aprender a ser madre necesitaste apoyo de tu familia. Ahora, para enfrentar una situación, ¿a quién le solicitarías apoyo? ¿De una pareja no? (...). (Familia monoparental)

Cuando me quedé embaraza, él no estaba, se había ido afuera, él no supo, no más vino a saber cuando el niño tenía como 19 años. 
Apoyo social para la parentalidad: experiencias en programas de infancia en Chile / Sanhueza et al.

De primera yo lloraba por el camino, porque no llegaba nunca, yo estaba embarazada de mi guagua y mi otro niño más chico tenía 4 años y tenía que darle pecho todavía y cambiarle paños. (Familia rural no mapuche)

Es sacrificado vivir aquí, él (familiar que cuenta con vehículo) no va a gastar bencina para llevarnos a nosotros. (Familia rural no mapuche)

\section{Redes de apoyo para la parentalidad}

Un 78,1\% reconoce contar principalmente con el apoyo de instituciones cuando enfrenta una dificultad en la crianza y un $64,3 \%$ destaca el establecimiento educacional como fuente de apoyo en tareas o responsabilidades escolares. Solo un $31 \%$ señala contar con amigos(as) ante la necesidad de recibir apoyo emocional, instrumental o informativo, y un 52\% expresa que, cuando necesita desahogarse o contar problemas que ha tenido para criar a sus hijos(as), acude a su familia y amigos. Los datos muestran que la contención familiar no es frecuente, ya sea por distanciamiento afectivo, conflictos familiares o lejanía, y solo un 52,6\% afirma contar con apoyo instrumental, que se refiere específicamente a recursos económicos entregados por el padre de los niños y niñas para solventar gastos que demanda la crianza.

El aislamiento social se agrava cuando los vínculos con la comunidad se encuentran deteriorados, especialmente en el caso de familias indígenas mapuches, para quienes la pertenencia cultural y territorial forma parte de su identidad. En ocasiones, esta desvinculación hacia la comunidad puede compensarse con una alta valoración del apoyo brindado por las redes formales, como los programas de protección de derechos dependientes del SENAME:

Desde que me quitaron a mis niños nunca más con la comunidad; yo no cuento con el apoyo de ellos, si me falta algo prefiero pedir ayuda afuera... cuando estaba viva mi mamita con ella los dejaba... cuando tenía que ir a hacer trámites... los dejaba con ella... ahora, gracias a ellos yo pude salir adelante... me orienté 
más con mis hijos... lo más importante es el SENAME... (Familia mapuche rural)

En cuanto a experiencias de apoyo social desde redes informales, se menciona:

En la iglesia sí, el apoyo de ellos es incondicional, los vecinos lo llevan a jugar con los niños. (Familia nuclear)

Aquí entre todos se cuidan, cuando salen a jugar, por ejemplo, a la pelota, el vecino, que tiene negocio, cuando viene un vehículo les dice. (Familia extensa)

Sin embargo, son otras mujeres quienes se constituyen en el mayor soporte emocional para las madres o cuidadoras:

Mi hija y mi mami siempre me apoyaron. Si fueran otras, me dirían "entrégalo no más, estas sufriendo mucho". No sabemos cómo va a salir, pero no, todos están conmigo. (Familia nuclear)

La familia es todo para mí... mi hermano, o amigas de repente. (Familia monoparental)

Mi pareja también, pero no es tanto como mi amiga. (Familia extensa)

Cuando tengo problemas me las aguanto sola, no converso con nadie, me encierro, prefiero estar sola. Escucho mi música, me fumo mi cigarrito, de repente viene para acá (amiga), conversamos, me cuenta sus cosas y yo les cuento las mías, así que con ella tenemos harta amistad, confianza. (Familia rural no mapuche)

En relación con el apoyo recibido de los programas de protección de derechos de infancia, las mujeres entrevistadas indican una diversidad de experiencias:

Nos hacen charlas de cómo tenemos que criarlos, de cómo comportarnos como mamás, la paciencia que hay que tener igual, me aconsejan, me ayudan. (Familia nuclear) 
Apoyo social para la parentalidad: experiencias en programas de infancia en Chile / Sanhueza et al.

A nosotros nos ha ayudado para guiar, porque de la fundación a ellos los citan también y les dan charlas y conversan con ellos. (Familia extensa)

No lo considero un apoyo. (Familia monoparental)

Le dije a la psicóloga el otro día, que ya no quería ir más, pero me dijo que no podía ser, porque esto era judicial y si yo lo abandono iba a ser culpa mía. (Familia rural no mapuche)

\section{Valoración de las redes de apoyo}

Las madres o cuidadoras identifican las redes formales de apoyo como aquellas más accesibles, ya sea porque están obligadas a participar en ellas o porque los profesionales las visitan periódicamente:

Nosotros no nos sentimos cómodos porque sentimos que nos están controlando y no respetan la confidencialidad, y ellas (trabajadoras sociales) se sienten con autoridad de entrar a la casa, que no se centren tanto en el problema. Nos gustaría que esto tuviera avances, creo que cambiarnos a otro programa es un retroceso. (Comentario escala folio 33)

Sin embargo, la valoración cualitativa muestra que el apoyo social brindado por los programas de protección de derechos de infancia no es suficiente. Más que potenciar los recursos de las familias, tiende a disminuir su autoeficacia, puesto que se privilegia el control y la vigilancia por sobre el apoyo social:

Más que apoyo familiar, hubo críticas por parte de los profesionales... que se mantenga la relación como familia, no que la destruyan los programas. (Comentario escala folio 38)

En los relatos de las mujeres a cargo de sus hijos o de otros niños $y$ niñas, se aprecia un bajo empoderamiento de su rol como madres o cuidadoras. Ellas sienten que aún tienen mucho que aprender y mejorar para ser "buenas madres", y que ese conocimiento se puede adquirir a través de talleres realizados por las profesionales ex- 
pertas, invisibilizando los saberes prácticos adquiridos mediante la experiencia y que pueden ser compartidos en espacios de intercambio horizontal con redes informales:

Me encantaría algún tipo de taller donde puedan orientar de mejor forma, para ser mejores padres. (Comentario escala folio 37)

Recibir mayor orientación sobre apoyo con nuestros nietos. (Comentario escala folio 2).

Yo, como mamá, pienso que hay muchas madres que están pasando momentos difíciles con sus hijos, que se sienten sobrepasados de trabajo y se frustran al no poder ayudar a sus hijos, $y$ callan sus penas a pesar de todo lo que tengan que hacer. Pero pienso que la ayuda podría ser más intensa como talleres. (Comentario escala folio 2)

En consecuencia, las capacidades siguen ancladas en los profesionales reconocidos como expertos, lo que puede generar, a largo plazo, la dependencia de parte de las madres o cuidadoras, y la cronificación del problema como una estrategia para mantener la relación de ayuda.

\section{Conclusiones}

Los programas de protección de derechos de infancia en Chile trabajan principalmente con mujeres, como principales figuras parentales en la vida de los niños y niñas. Los programas buscan fortalecer a estas mujeres, sean madres, abuelas, tías, nuevas parejas del padre o simplemente cuidadoras, atribuyéndoles la responsabilidad primordial del bienestar de los niños y las niñas, y perpetuando el "contrato sexual" que relega a la mujer al espacio privado, las tareas domésticas y el cuidado de los otros. Se constata que los programas de protección de derechos de infancia perpetúan el estereotipo de género que vincula a la mujer con la maternidad y le asigna el rol de cuidadora principal, a pesar de su creciente incorporación al mundo laboral (Cerruti y Binstock, 2009; Arias, Saavedra y Avilés, 2017). 
Apoyo social para la parentalidad: experiencias en programas de infancia en Chile / Sanhueza et al.

Las dificultades y necesidades de apoyo frente a la parentalidad son múltiples. Destacan las de tipo emocional — sentimientos de angustia y frustración que se viven en el proceso de crianza-; las instrumentales - escasez de recursos económicos-, y las informativas - desconocimiento de cómo abordar algunos aspectos del cuidado. Se reporta la presencia de redes de apoyo formales e informales, en las que priman las primeras. Las figuras parentales tienden a buscar el apoyo emocional en sus redes informales, familia y amigos, lo que responde a la necesidad natural de ser acompañados por otros que hayan vivido situaciones similares (Gracia, 1997). Las instituciones del Estado constituyen la principal fuente de apoyo instrumental, ya que entregan recursos económicos para cubrir las necesidades de los niños y las niñas. En este ámbito, la familia extensa aparece más relegada, lo que probablemente se deba a que también enfrenta dificultades económicas en su vida cotidiana y no cuenta con los recursos para brindar este apoyo. La necesidad de apoyo informativo tiende a darse en el ámbito educativo y de salud, donde surgen temas que las madres reconocen ignorar y en los que no reciben ni la orientación necesaria ni la información clara. Que las figuras parentales no reciban información suficiente o no logren comprenderla tal como es entregada puede tener efectos nocivos en el proceso de crianza del niño, por ejemplo, en el ámbito de la salud. Por otra parte, cuando las madres cuentan con un menor nivel educacional que sus hijos, las posibilidades de acompañarlos en su proceso educativo se reducen y aumenta el rezago escolar o las brechas de aprendizaje de estas niñas y niños, cuando no existe un apoyo externo.

La situación de las madres o cuidadoras que enfrentan en solitario la tarea parental es particularmente crítica cuando no cuentan con redes de apoyo social. Esto no se debe a la estructura familiar, sino a la situación de aislamiento social en que se encuentran algunas de estas mujeres y a las altas exigencias que implica la parentalidad, ya que no cuentan con el apoyo esperado, especialmente en lo emocional. Esta situación puede llevar a estas mujeres a experimentar, a largo plazo, serios problemas de salud mental, que 
repercutirán en la calidad de la relación y en el cuidado hacia los niños y las niñas. En este sentido, el Trabajo Social tiene un papel clave, al desentrañar las necesidades de las madres o cuidadoras. Las estrategias de intervención centradas exclusivamente en el niño no apuntan a fortalecer la relación parental, sino que minimizan o invisibilizan las dificultades que las propias madres enfren$\tan$ (Turney, 2012; Featherstone, White \& Morris, 2014) y, por lo tanto, no se activan los apoyos necesarios para afrontar dichas dificultades, lo que puede conllevar un fracaso de la intervención.

Por otra parte, las madres indígenas viven una doble situación de aislamiento social cuando son rechazadas por su comunidad de origen y tienden a estrechar vínculos con redes formales fuera de su entorno. El apoyo social que pueden brindar estas redes es fundamental y altamente valorado; sin embargo, si la intervención no se orienta también a recomponer los vínculos de estas madres con sus redes informales en el entorno comunitario más cercano, es probable que se generen relaciones de dependencia con las redes formales y una cronificación de los procesos de intervención. La expectativa del término de la intervención puede ser vivida como una grave pérdida de apoyo, sensación de soledad e incertidumbre de las propias capacidades para enfrentar en el futuro las tareas parentales. Esto último repercute negativamente en la eficacia parental y, por lo tanto, en el grado de satisfacción que pueden llegar a sentir estas madres en la relación con sus hijos.

Las madres o cuidadoras que se relacionan mayoritariamente con redes formales pueden ver deteriorada su autoeficacia parental, generando dependencia y cronificación de la relación con los y las profesionales, puesto que las redes formales legitiman el saber experto mediante categorías como "competencias parentales", "pautas de crianza", por oposición a la sabiduría práctica que se construye en la experiencia cotidiana de las madres en sus propios contextos.

En general, la autoeficacia parental es poco cuidada en los procesos de intervención de los programas de protección de infan- 
cia. La madres y cuidadoras señalan sentimientos de devaluación, coincidentemente con las experiencias de padres y madres del Reino Unido, atendidos en programas similares (Rorty, 1989). Asimismo, esta situación de menoscabo (Smithson \& Gibson, 2017) se agrava cuando se trata de mujeres, dado que son ellas quienes afrontan las expectativas culturales que sacralizan la maternidad (Badinter, 1981), y exigen respuestas frente a necesidades de los niños y las niñas a su cuidado, quienes, en situaciones de aislamiento social y falta de apoyo emocional de parte de redes informales, superan sus posibilidades.

Finalmente, es necesario que las redes formales de apoyo tengan en el horizonte de sus intervenciones un enfoque de género, que reconozca la presencia mayoritaria de mujeres en sus programas e incentive la incorporación de las figuras parentales masculinas, con el fin de tener una parentalidad compartida. Es necesario, también, generar espacios de contención y orientación más horizontales, que brinden la posibilidad de compartir experiencias con otras figuras parentales que están enfrentando las mismas dificultades, con el objeto de evitar la sensación de aislamiento social y activar redes informales de apoyo ante las dificultades de la parentalidad, que sean permanentes en la vida de las personas después de los procesos de intervención institucional.

\section{Bibliografía}

Aguayo, F., Barker, G. y Kimelman, E. (2016). Paternidad y Cuidado en América Latina: Ausencias, Presencias y Transformaciones. Masculinities and Social Change, 5(2), 98-106. DOI:10.17583/MCS.2016.2140

Ayala-Nunes, L., Lemos, I. \& Nunes, C. (2013). Predictores del estrés parental en madres de familias en riesgo psicosocial. Universitas Psychologica, 13(2), 529-539. Recuperado a partir de https://revistas.javeriana.edu.co/index.php/revPsycho/ article/view/4151

Arias, S., Saavedra, F. \& Avilés, I. (2017). El cuidado: una actividad de riesgo en tiempos de crisis. Una revisión de la investigación con cuidadoras españolas. Psicoperspectivas, 16(1), 42-54. DOI: http://dx.doi.org/10.5027/psicoperspectivasvol15-issue 3 -full-796 
Badinter, E. (1981). ¿Existe el instinto maternal? Historia del amor maternal. Siglos XVII al XX. Barcelona, España: Paidós Ibérica. Recuperado de http://bibliotecafeminista.com/existeamor-maternal-historia-del-amor-maternal-siglos-xvii-xx/

Bardin, L. (1986). Análisis de Contenido. Madrid: Ediciones Akal. S. A.

Beauvoir, S. (1949). El segundo sexo. Buenos Aires: Siglo Veinte.

Berger, P. \& Luckmann, T. (2008). La construcción social de la realidad. Buenos Aires: Amorrortu Editores.

Bravo, A. \& Del Valle, J. (2003). Las redes de apoyo social de los adolescentes acogidos en residencias de protección. Un análisis comparativo con población normativa. Psicothema, 15, 136-142. Recuperado de http://www.redalyc.org/articulo. oa? id $=72715122$

Bourdieu, P. (1997). El espíritu de familia. Razones prácticas. Sobre la teoría de la acción. Barcelona: Anagrama.

Burín, M. \& Meler, I. (2001). Género y familia: poder, amor y sexualidad en la construcción de la subjetividad. Buenos Aires: Paidós.

Cabrolié, M. \& Sanhueza, L. (2016). Una mirada crítica desde el Trabajo Social chileno a la política pública de infancia: El enfoque de condiciones en la evaluación de la parentalidad, en M. De Martino y V. Giorgi (eds.), Miradas iberoamericanas sobre prácticas profesionales en el campo familiar (pp. 5572). Montevideo: Editorial EPPAL.

Cerruti, M. \& Binstock, G. (2009). Familias latinoamericanas en transformación: desafíos y demandas para la acción pública. Serie Políticas Sociales N¹47. CEPAL. División de Desarrollo Social. Santiago de Chile: Naciones Unidas. Recuperado de http://repositorio. cepal.org/bitstream/handle/11362/6153/S0900608_ es.pdf? sequence $=1$ \&isAllowed $=\mathrm{y}$.

Correa, N., Rodrigo, M., Rodríguez, M., \& Simón, M. (1998). Desarrollo y educación familiar en niños con cursos evolutivos diferentes. En M. Rodrigo y J. Palacios (eds.), Familia y Desarrollo humano (pp. 445-464). Madrid: Alianza Editorial.

Chacón, F. \& Tapia, M. (2017). No quiero tener hijos (as)... continuidad y cambio en las relaciones de pareja de mujeres profesionales jóvenes, Polis [En línea], 46, Publicado el 12 junio 2017, recuperado el 16 junio 2017 de http://polis.revues. org/12339

Daining, C. \& De Panfilis, D. (2007). Resilience of youth in transition from out-of-home care to adulthood. Children and Youth Services Review, 29, 1158-1178. DOI: https://doi. org/10.1016/j.childyouth.2007.04.006 
Apoyo social para la parentalidad: experiencias en programas de infancia en Chile / Sanhueza et al.

Díaz, A., Pérez, J., Martínez, M. T., Brito, A. \& Herrera, E. (2001). El vínculo de apego en bebés prematuros: incubadora y método canguro. Revista de Atención Temprana, 4(1), 11-19.

Farkas, C. (2008). Escala de Evaluación Parental (EEP): Desarrollo, propiedades psicométricas y aplicaciones. Universitas Psychologica, 7(2), 457-467. Recuperado de http://www.scielo. org.co/pdf/rups/v7n2/v7n2a12.pdf

Featherstone, B., White, S. \& Morris, K. (2014). Re-Imagining Child Protection - Towards Humane Social Work with Families. Bristol: Policy Press.

Friedan, B. (2009). La mística de la feminidad. España: Ediciones Cátedra.

García, B. \& De Oliveira, O. (2011). Cambios familiares y políticas públicas en América Latina. México: Centro de Estudios Demográficos, Urbanos y Ambientales y Centro de Estudios Sociológicos de El Colegio de México. Recuperado de https://www.annualreviews.org/doi/pdf/10.1146/annurev-soc-033111-130034.

Gallardo, L. \& Gálvez, L. (2018). Validación del cuestionario de apoyo social comunitario en personas mayores chilenas. Revista Iberoamericana de diagnóstico y Evaluación Psicológica, 4(49), 47-57.

Gómez, E., Muñoz, M. \& Haz, A. M. (2007). Familias multiproblemáticas y en riesgo social: características e intervención. Psykhe (Santiago), 16(2), 43-54. DOI: https://dx.doi.org/10.4067/ S0718-22282007000200004

Gracia, E. (1997). El apoyo social en la intervención comunitaria. Barcelona: Paidós.

Gracia, E., Herrero, J. \& Musitu, G. (2002). Evaluación de recursos y estresores psicosociales en la comunidad. Madrid: Síntesis.

Jociles, M. I., Rivas, A. M., Moncó, B., Villamil, F. \& Díaz, P. (2008). Una reflexión crítica sobre la monoparentalidad: el caso de las madres solteras por elección. Revista Portularia, 7(1), 265-274. Recuperado de http://rabida.uhu.es/dspace/bitstream/handle/10272/2186/b15506010.pdf

Kendrick, A. (2005). Social exclusion and social inclusion: Themes and issues in residential child care, en D. Crimmens e I. Milligan (eds.), Facing forward. Residential child care in the 21st century (pp. 7-18). Dorset: Russel House Publishing.

Knibiehler, Y. (2001). La construction sociale de la maternité. En Y. Knibiehler (Ed.), Maternité, affaire privée, affaire publique (pp. 387-393). Paris: Bayard. 
Martín, E. \& Dávila, L. (2008). Redes de apoyo social y adaptación de los menores en acogimiento residencial. Psicothema, 20, 229-235. Recuperado de http://www.psicothema.com/ pdf/3453.pdf

Martin, E. (2011). Apoyo social percibido en niños y adolescentes en acogimiento residencial. International Journal of Psychology and Psychological Therapy, 11(1), 107-120. Recuperado de http://www.ijpsy.com/volumen11/num1/285/apoyosocial-percibido-en-nios-y-adolescentes-ES.pdf

Meler, I. (1998). Amor y convivencia entre los géneros a fines del siglo XX, en M. Burin e I. Meler. Género y familia. Buenos Aires: Paidós.

Navarro, P. \& Díaz, C. (1995). Análisis de Contenido, en J. Delgado y J. Gutiérrez (eds.), Métodos y técnicas cualitativas de investigación en ciencias sociales (pp. 177-224). España: Editorial Síntesis.

Olhaberry, M. \& Farkas, C. (2012). Estrés Materno y Configuración Familiar: Estudio comparativo en Familias Chilenas Monoparentales y Nucleares de bajos ingresos. Universitas Psychologica, 11(4), 1326. DOI: https://doi.org/10.11144/Javeriana.upsy11-4.emcf

Piñuel, J. (2002). Epistemología, metodología y técnicas del análisis de contenido. Estudios de Sociolingüística, 3(1), 1-42. Recuperado de https://www.ucm.es/data/cont/docs/268-201307-29-Pinuel_Raigada_AnalisisContenido_2002_EstudiosSociolinguisticaUVigo.pdf

Pozo Cabanillas, P., Sarriá Sánchez, E. \& Méndez Zaballos, L. (2006). Estrés en madres de personas con trastornos del espectro autista. Psicothema, 18(3), 342-347

Rodrigo, M. (2009). Una mirada integradora de la resiliencia parental: desde el contexto hasta la mente de las madres y los padres en riesgo psicosocial. Psicologia da Educação, 28, 51-71. Recuperado de http://pepsic.bvsalud.org/pdf/psie/n28/ v28a04.pdf

Rodrigo, M., Márquez, M. \& Martín, J. (2010). Parentalidad positiva y políticas locales de apoyo a las familias. Orientaciones para favorecer el ejercicio de las responsabilidades parentales desde las corporaciones locales. Madrid: Federación Española de Municipios y Provincias (FEMP). Recuperado de https:// www.msssi.gob.es/ssi/familiasInfancia/docs/folletoParentalidad.pdf.

Rorty, R. (1989). Contingency, Irony, and Solidarity. Cambridge: Cambridge University Press. 
Apoyo social para la parentalidad: experiencias en programas de infancia en Chile / Sanhueza et al.

Schütz, A. (2003) El problema de la realidad social. Escritos I. Buenos Aires: Amorrortu Editores.

Strauss, A. \& Corbin, J. (2002). Bases de la investigación cualitativa: técnicas y procedimientos para desarrollar la teoría fundamentada. Bogotá: Universidad de Antioquia.

Smithson, R. \& Gibson, M. (2017). Less than human: a qualitative study into the experience of parents involved in the child protection system. Child and Family Social Work, 22, 565-574. DOI:10.1111/cfs.12270.

Terol M., López S, Neipp, M., Rodríguez, J., Pastor, M. \& MartínAragón, M. (2004). Apoyo social e instrumentos de evaluación: revisión y clasificación. Anuario de Psicología, 35, 23-45. Recuperado de https://dialnet.unirioja.es/servlet/ articulo? codigo $=864595$

Turney, D. (2012). A relationship-based approach to engaging involuntary clients: the contribution of recognition theory. Child and Family Social Work, 17(2), 145-159. DOI: https://doi. org/10.1111/j.1365-2206.2012.00830.x 